\title{
HYBRID METHODS FOR STUDYING STABILITY AND BIFURCATIONS IN DELAYED FEEDBACK SYSTEMS
}

\author{
GRISELDA R. ITOVICH ${ }^{*}$ \\ Escuela de Producción, Tecnología y Medio Ambiente, \\ Sede Alto Valle y Valle Medio, Universidad Nacional de Río Negro \\ R8336ATG Villa Regina, Argentina \\ FRANCO S. GENTILE \\ Departamento de Matemática, Universidad Nacional del Sur \\ Instituto de Investigaciones en Ingeniería Eléctrica - IIIE (UNS - CONICET) \\ B8000CPB Bahía Blanca, Argentina \\ JORGE L. MOIOLA \\ Departamento de Ingeniería Eléctrica y de Computadoras, Universidad Nacional del Sur \\ Instituto de Investigaciones en Ingeniería Eléctrica - IIIE (UNS - CONICET) \\ B8000CPB Bahía Blanca, Argentina \\ Received (to be inserted by publisher)
}

\begin{abstract}
The dynamics of two related models of second order delay differential equations with four bifurcating parameters are analyzed. Through a classical technique in the time domain which involves the location of the roots of an exponential polynomial equation, the areas of stability of the equilibrium are set. A frequency-domain methodology is applied to study the Hopf bifurcation phenomena and to describe the behavior of the emerging cycles completely via a feedback system approach. Certain type of singularities, which provoke fold bifurcations of cycles are detected precisely. Also, a complete picture of parameter configurations to produce resonances is established for both models. The whole results are checked with the software DDE-Biftool.
\end{abstract}

Keywords: delay differential equations, stability analysis, bifurcations, limit cycles, resonances

\section{Introduction}

The estimation of the critical gain and the critical frequency in feedback control systems has a very long tradition as limiting cases in engineering systems. In this regard, great efforts have been employed to use simple methods in order to handle the stability issues and, at the same time, to improve the automatic tuning of simple regulators [Åström \& Hägglund, 1984; Schei, 1994]. The computation of the oscillation characteristics has been obtained using the approach of relay systems and/or the describing function analysis [Atherton, 1975] in order to set later the parameters of the proportionalintegral-derivative (PID) controllers under specified rules. In particular, autotuning principles for generalization of PID controllers have been applied also to SISO (single-input to single-output) systems with delays (see, for example, Prokop et al. [2012] and the references therein).

\footnotetext{
*E-mail: gitovich@unrn.edu.ar
} 
The determination of the critical gain and the critical frequency, simultaneously, plays a singular role for stability issues when dealing with a nonlinear feedback system since autonomous oscillations are expected to arise under the now classical phenomenon of Hopf bifurcation. A timely approach suitable for control engineering has been presented in Mees and Chua [1979] and Mees [1981] inspired from previous results given by the describing function and harmonic balance analyses [Allwright, 1977]. A rather similar scenario had been announced before for special systems having a timedelayed feedback as it was shown in the pioneering work of Tsypkin [1946]. That contribution was certainly proposed very early compared now with other methods for analyzing the stability of retarded functional differential equations. Then, by using the framework of Hopf bifurcation in control systems given in Mees [1981], the result of Tsypkin [1946] has been reinforced in the work of Moiola and Chen [1996] and Gentile et al. [2012]. In this regard, the frontiers of stability are called Hopf bifurcation curves in the space of system parameters. Moreover, taking into account the complexity added by the delays, the characteristic polynomial is now changed to an exponential polynomial as presented in the now classical works of Bellman and Danskin [1954] and Bellman and Cooke [1963]. The complexity of stability is enlarged due to the possibility of multiple root crossing as it was shown in a clever tutorial by Sipahi et al. [2011] where several recent methodologies are placed together in attractive and useful control engineering terms. This is the case of the appearance of resonances since two critical frequencies coincide for the same values of system parameters, as it was shown previously using tools from bifurcation theory [Campbell \& Bélair, 1999].

In the present article, the analysis of the Hopf bifurcation condition and the regions of stability using a type of hybrid methods complemented with the frequency-domain approach [Mees, 1981; Moiola $\&$ Chen, 1996] is presented. Two models of second order delay differential equations involving the variation of four parameters are studied. The results are complementary of those recently shown in Gentile et al. [2018] in which an analytical method plus a numerical computational one provided by DDEBiftool (see Engelborghs et al. [2002]) have been successfully applied.

From now on, the main results are presented in the following two sections. In Section 2, the two models are exposed and the stability of the equi- librium is examined via a time-domain technique. Then, in Section 3 both models are further analyzed in the frequency-domain setting. The Hopf bifurcation phenomena is considered, the stability of the emergent orbits is fully determined and some degeneracies are found. At last, some conclusions are given.

\section{Delay Models and Equilibrium Stability}

The aim of this work is the analysis of the dynamics of the following delay-differential equation

$$
\ddot{x}+\gamma x=\alpha u+\beta u^{2},
$$

where $\dot{x}=\frac{d x}{d t}, \gamma>0, \alpha, \beta \neq 0$, for two particular models, where $u=x_{\tau}$ or $u=\dot{x}_{\tau}$ with $x_{\tau}=x(t-\tau)$, $\tau>0$. One specific case with $u=x_{\tau}$ has been considered by Campbell and LeBlanc [1998] in relation with the study of the 1:2 resonance and its treatment by normal forms. The most general situation has been considered in Campbell and Bélair [1999] where the action of a restoring force depends on the velocity $\dot{x}$. In this work, the model (1) with four parameters (counting the delay $\tau$ ), is explored with different techniques to discover and clarify some inherent dynamic aspects.

To analyze the stability of one equilibrium point of (1), it is necessary to find the location of the roots of a certain exponential polynomial. In this regard, the following definitions and results will be used.

Definition 2.1. Let $p(x, y)$ be a two variable polynomial. Then $P(z)=p\left(z, e^{z}\right)=\sum_{m, n} a_{m n} e^{z m} z^{n}$ is an exponential polynomial.

Definition 2.2. Let $P(z)$ be an exponential polynomial. The term $a_{r s} e^{z r} z^{s}$ is called the principal term of $P$ if $a_{r s} \neq 0$ and, if for each other term $a_{m n} e^{z m} z^{n}$ with $a_{m n} \neq 0$, it is satisfied $r>m, s>n$, or $r=m, s>n$, or $r>m, s=n$.

Remark 2.1. It can be proved that if an exponential polynomial $P(z)$ has no principal term then it has an unbounded number of zeros with arbitrarily large real part [Bellman \& Danskin, 1954; Bellman $\&$ Cooke, 1963], e.g. $P(z)=e^{z}-z$.

The next results [Bellman \& Danskin, 1954; Pontryagin, 1955; Bellman \& Cooke, 1963] are required for the forthcoming stability analysis. Let $P=P(z)$ be an exponential polynomial whose 
coefficients are real with a principal term, where $P(i y)=F(y)+i G(y)$. It can be easily proved that $F$ results an even function whereas $G$ is an odd function.

Notation: The expression $F G^{\prime}(y)$ means $F(y) G^{\prime}(y)$. The same sense holds for $F^{\prime} G(y)$.

Theorem 1. If all the zeros of $P$ are located on the left half plane then all the zeros of $F$ and $G$ are real, alternating and $F G^{\prime}(y)-F^{\prime} G(y)>0$, for all real value $y$.

Theorem 2. All the zeros of $P$ are located on the left half plane if

I) all the roots of $F$ are real and for each of these zeros the condition $F^{\prime} G(y)<0$ is satisfied, or

II) all the roots of $G$ are real and for each of these zeros the condition $F G^{\prime}(y)>0$ is satisfied.

Remark 2.2. Notice that the condition of $F(y)$ and $G(y)$ to be simultaneously zero is equivalent to state that $P$ has a pair of pure imaginary roots.

\subsection{Model 1}

Consider (1) with $u=x_{\tau}$, that can be written as

$$
\dot{x}_{1}=-\gamma x_{2}+\alpha x_{2}(t-\tau)+\beta x_{2}^{2}(t-\tau), \quad \dot{x}_{2}=x_{1},
$$

and its steady states are $X_{1}=(0,0)$ and $X_{2}=$ $\left(0,(\gamma-\alpha) \beta^{-1}\right)$. If $\gamma=\alpha$, then a transcritical bifurcation of equilibria is observed. To avoid this particular condition, in the rest of the paper it is assumed that $\gamma \neq \alpha$.

For the stability analysis of each equilibrium $X_{i}, i=$ 1,2 , the linearization of (2) about $X_{i}$ must be considered. From (2), only for the case of $X_{1}$ results

$$
\dot{c}=A c+A_{\tau} c(t-\tau),
$$

where

$$
A=\left(\begin{array}{cc}
0 & -\gamma \\
1 & 0
\end{array}\right) \text { and } A_{\tau}=\left(\begin{array}{ll}
0 & \alpha \\
0 & 0
\end{array}\right)
$$

The characteristic equation $\operatorname{det}\left(\lambda I-A-A_{\tau} e^{-\lambda \tau}\right)=$ 0 , after changing variables as $z=\lambda \tau$, becomes

$$
P_{1}(z)=p\left(z, e^{z}\right)=e^{z}\left(z^{2}+\gamma \tau^{2}\right)-\alpha \tau^{2}=0 .
$$

The principal term of $P_{1}$, according with Definition 2 , is $e^{z} z^{2}$. Then, by (4) it is written $P_{1}(i y)=$ $F_{1}(y)+i G_{1}(y)$ which yields

$$
\begin{aligned}
& F_{1}(y)=\left(-y^{2}+\gamma \tau^{2}\right) \cos y-\alpha \tau^{2}, \\
& G_{1}(y)=\left(-y^{2}+\gamma \tau^{2}\right) \sin y .
\end{aligned}
$$

The detailed work that follows aims to prove that all the roots of $P_{1}(z)$ have negative real part under certain parameter constraints. This fact guarantees the asymptotic stability of the equilibrium point $X_{1}$. In order to apply Theorem 2 , it is necessary to set conditions on the parameters to satisfy the required hypotheses. Observe that the roots of $G_{1}$ are
a) $y_{k}=k \pi, k \in \mathbb{Z}$,
b) $\hat{y}_{1,2}= \pm \sqrt{\gamma} \tau$.

Remark 2.3. To satisfy the condition II) $\left(F_{1} G_{1}^{\prime}(y)>\right.$ 0 ) of Theorem 2, it is necessary that $F_{1}(y) \neq 0$ for each root of $G_{1}$, which in addition must be all simple (to carry out $G_{1}^{\prime}(y) \neq 0$ ).

Then, due to (6):

a) It is precise that $F_{1}\left(y_{k}\right) \neq 0$, so considering (5) the parameters $(\gamma, \tau, \alpha)$ should not satisfy the condition

$$
(-1)^{k}\left(-y_{k}^{2}+\gamma \tau^{2}\right)-\alpha \tau^{2}=0, \quad k \in \mathbb{Z} .
$$

b) It is always true that $F_{1}\left(\hat{y}_{1,2}\right)=-\alpha \tau^{2} \neq 0$, due to $\alpha, \tau$ are nonzero.

c) $\mathrm{As}$

$$
G_{1}^{\prime}(y)=-2 y \sin y+\left(-y^{2}+\gamma \tau^{2}\right) \cos y,
$$

then it is also required that the parameters do not check

$$
\gamma \tau^{2}=y_{k}^{2}=k^{2} \pi^{2}, k \in \mathbb{Z},
$$

to fulfill $G_{1}^{\prime}(y) \neq 0$, for any root $y$ of $G_{1}$.

From now on, it will be considered $\gamma=1$. Actually, (1) can be reduced to a three-parameter model changing the time scale as $t^{\prime}=\sqrt{\gamma} t$. So, in the following results the assumption $\gamma=1$ does not mean a loss of generality. Due to (9), two situations for the parameter $\tau$ will be considered: 1) $0=y_{0}<\tau<y_{1}$ or 2) $y_{k}<\tau<y_{k+1}, k \in \mathbb{N}$.

Theorem 3. Consider (4) and (5) with $\tau>0, \alpha \neq$ 0,1 , together with $y_{k}, k \in \mathbb{N}^{0}$ and $\hat{y}_{1,2}$ given by (6). Assume that the parameter values do not satisfy neither (7) nor (9). All the roots of $P_{1}$ lie on the left half plane if and only if the following parameter conditions are fulfilled:

I) For $0<\tau<y_{1}$, it must be

$$
\alpha<\frac{y_{1}^{2}}{\tau^{2}}-1, \quad \alpha<1, \quad \alpha>0 .
$$

II) For $y_{k}<\tau<y_{k+1}, k \in \mathbb{N}$, it is required:

a) If $k$ is odd, then

$$
\alpha>\frac{y_{k}^{2}}{\tau^{2}}-1, \quad \alpha>-\frac{y_{k+1}^{2}}{\tau^{2}}+1, \quad \alpha<0 .
$$


b) If $k$ is even, then

$$
\alpha<-\frac{y_{k}^{2}}{\tau^{2}}+1, \quad \alpha<\frac{y_{k+1}^{2}}{\tau^{2}}-1, \quad \alpha>0 .
$$

Proof. $\Longrightarrow$ (Necessary condition) This part follows using Theorem 1. It is required that the zeros of $F_{1}$ and $G_{1}$ must be real, so an adequate result is used to prove this statement [Bellman \& Cooke, 1963]. It is clear that all the zeros of $G_{1}$ (6) are real and effectively there are $4 k+2$ in any interval $[-2 k \pi+\pi / 2,2 k \pi+\pi / 2]$ which includes $\tau$. In order to assert that the roots of $F_{1}$ are real (see (5)), it is analyzed the equation $\cos y=\alpha \tau^{2} /\left(-y^{2}+\tau^{2}\right)$. In the sequel and due to the parity, it must be proved that there are $2 k+1$ roots in some interval $[0,2 k \pi]$ for $k$ sufficiently large.

I) Suppose $0<\tau<y_{1}=\pi$. It is considered first the interval $(0, \tau)$. The roots of $G_{1}(6)$ should alternate with $F_{1}$ 's by Theorem 1 . As $F_{1}(0)=$ $(1-\alpha) \tau^{2}, F_{1}(\tau)=-\alpha \tau^{2}$ then $F_{1}(0)$ and $F_{1}(\tau)$ have different sign only if $0<\alpha<1$. By Bolzano's theorem $F_{1}$ has a root in $(0, \tau)$ and results $F_{1}(\tau)<0$. Now, considering the zeros of $G_{1}$ and the interval $\left(\tau, y_{1}\right)$ it follows that $F_{1}\left(y_{1}\right)=\left(-y_{1}^{2}+\tau^{2}\right)(-1)-\alpha \tau^{2}$ should be positive. This means $\alpha<y_{1}^{2} / \tau^{2}-1$. Thus two roots of $F_{1}$ exist in $[0, \pi]$.

Now, let be $0<\tau<y_{1}<y_{j}$. For $j$ odd, $j>$ $1, F_{1}\left(y_{j}\right)=\left(-y_{j}^{2}+\tau^{2}\right)(-1)-\alpha \tau^{2}>0$, which is equivalent to $\alpha<y_{j}^{2} / \tau^{2}-1$. This result is true under $\alpha<y_{1}^{2} / \tau^{2}-1$.

For $j$ even, $F_{1}\left(y_{j}\right)=\left(-y_{j}^{2}+\tau^{2}\right)-\alpha \tau^{2}<0$, which is equivalent to $\alpha>-y_{j}^{2} / \tau^{2}+1$. This assertion is valid under $0<\alpha<1$ due to $-y_{j}^{2} / \tau^{2}+1<0$ if $0<\tau<y_{1}<y_{j}$.

So in each interval $[(j-1) \pi, j \pi]$, where $j \geq 2, F_{1}$ has one root. Thereby, one has $2 k+1$ roots of $F_{1}$ in $[0,2 k \pi]$, which proves that they are all real.

II) The proof for Case IIa) appears in Appendix A. The proof for the remaining Case IIb) is analogous.

$\Longleftarrow$ (Sufficient condition, sketch) In order to apply Theorem $2 \mathrm{II}$ ), it is necessary to check that $F_{1} G_{1}^{\prime}(y)>0$ for each $y$, an arbitrary root of $G_{1}$ (see (6)).

I) The sign of $\alpha$ follows from the requirement of $F_{1} G_{1}^{\prime}\left(\hat{y}_{1,2}\right)>0$. The condition $\alpha<1$ results from the condition $F_{1} G_{1}^{\prime}\left(y_{0}\right)>0$. The main inequality is established by setting $F_{1} G_{1}^{\prime}\left(y_{1}\right)>0$. To satisfy $F_{1} G_{1}^{\prime}\left(y_{k}\right)>0, k \geq 2$, it can be shown that the last condition is sufficient, by considering separately the cases $k$ odd or even.
II) The sign of $\alpha$ is established from $F_{1} G_{1}^{\prime}\left(\hat{y}_{1,2}\right)>0$, according to $k$ being odd or even. The first two inequalities in a) and b) are deduced imposing $F_{1} G_{1}^{\prime}\left(y_{k}\right)>0$ and $F_{1} G_{1}^{\prime}\left(y_{k+1}\right)>0$, when $k$ is odd or even. These conditions result sufficient to guarantee $F_{1} G_{1}^{\prime}\left(y_{i}\right)>0$, where $i<k$ or $i>k+1$. Finally, to show this last it is necessary to consider the four different cases which result for $i$ odd or even. The details to prove the Case I and Case II)a) appear in Appendix A. The left over case is similar.

It still remains to test that $F_{1} G_{1}^{\prime}\left(y_{k}\right)>0$ for each $y_{k}$, a root of $G_{1}$ where $y_{k}<0$. Due to $F_{1} G_{1}^{\prime}$ is an even function, i.e. $F_{1} G_{1}^{\prime}\left(-y_{k}\right)=F_{1} G_{1}^{\prime}\left(y_{k}\right)$, now the proof is complete.

Corollary 2.1. The equilibrium $X_{1}$ in system (2) results asymptotically stable under the necessary and sufficient conditions established in Theorem 3.

Remark 2.4. Theorem 3 can be stated for any $\gamma>0$ in a similar way and analogous results can be formulated for the non trivial equilibrium of system (2).

Remark 2.5. A different proof of Theorem 3 has also been obtained through frequency-domain methods, more specifically by using the Nyquist stability criterion [Itovich et al., 2018].

Remark 2.6. The stability analysis for an equation like (1) with $u=x_{\tau}$, which includes a nonzero term in $\dot{x}$ in its left member can be found in Bellman and Cooke [1963].

Remark 2.7. When (7) is satisfied, the roots of (4) are on the imaginary axis. This condition determines the Hopf surfaces in the three dimensional parameter space $\gamma-\tau-\alpha$ (for system (2)). Or, fixing the variable $\gamma$, the Hopf curves $\alpha_{k}, k \geq 1$ in the $\tau-\alpha$ plane are determined by

$$
\alpha=\alpha_{k}(\tau)=(-1)^{k}\left(-\frac{y_{k}^{2}}{\tau^{2}}+\gamma\right) .
$$

Due to Theorem 3, Fig. 1 shows a few colored stability areas, some Hopf curves $\alpha_{k}(10)$ and multiple resonant points in the $\tau-\alpha$ plane, with $\gamma=1$. In this particular case, the curves $\alpha_{k}$ intersect the $\tau$ axis $(\alpha=0)$ at the points $\tau=y_{k}$. The curves $\alpha_{k}$ and $\alpha_{j}(k<j)$ intersect each other only if $k$ and $j$ have different parity. These intersections determine $k: j$ resonant points whose coordinates are

$$
(\tau, \alpha)=\left(\sqrt{\frac{y_{k}^{2}+y_{j}^{2}}{2}},(-1)^{k}\left(\frac{y_{j}^{2}-y_{k}^{2}}{y_{k}^{2}+y_{j}^{2}}\right)\right) .
$$




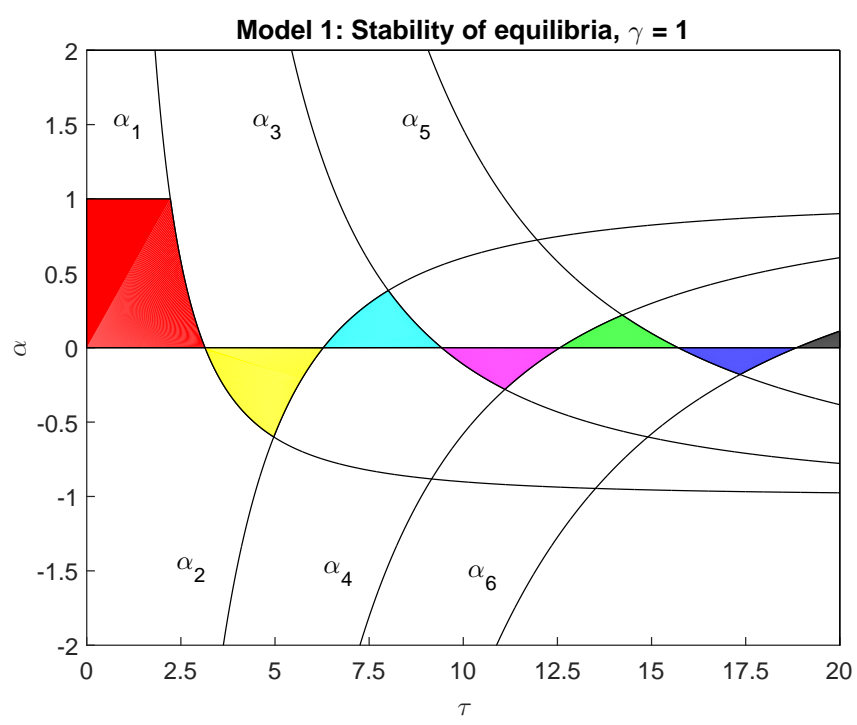

Fig. 1. Stability regions for the trivial equilibrium of system (2) with $\gamma=1$ and arbitrary $\beta$. According to Theorem 3 , regions in yellow, magenta and blue correspond to $k=1,3,5$, respectively, of situation (II)a), while areas in cyan, green and black (partially drawn) refer to $k=2,4,6$, under situation (II)b) and at last the red domain is related with the situation I).

At the resonance points, (4) has two pair of purely imaginary solutions, namely $\pm i y_{k} / \tau, \pm i y_{j} / \tau$ satisfying $y_{k} / y_{j}=k / j$. This fact is related in (2) with the interaction between two limit cycles, whose frequencies are $k \pi / \tau$ and $j \pi / \tau$ approximately. Thereby, the intersection between the Hopf curves $\alpha_{1}$ and $\alpha_{2}$ results in a $1: 2$ resonant point [Gentile et al., 2018].

When the initial segment of the static bifurcation line $\alpha=1$ crosses the Hopf curve $\alpha_{1}$ then a Gavrilov-Guckenheimer singularity appears, whose coordinates are $(\pi / \sqrt{2}, 1)$. This situation is repeated when $\alpha=1$ intersects the Hopf curve $\alpha_{k}$ where $k$ is odd and $k>1$.

Remark 2.8. A result equivalent to Theorem 3 can be established by the assignment of a fixed value of $\tau$ in (4) and (5). In this case, the resulting restrictions between $\gamma$ and $\alpha$ are always linear. Related with this assumption, the complete stability analysis of a general delay differential equation which includes (3) can be found in Stépán [1989]. Considering $\tau=\pi$, the Hopf curves of system (2) result $\bar{\alpha}_{k}=(-1)^{k}\left(-k^{2}+\gamma\right)$, where $k \in \mathbb{N}$, and the stability regions for the trivial equilibrium are shown in Fig. 2.

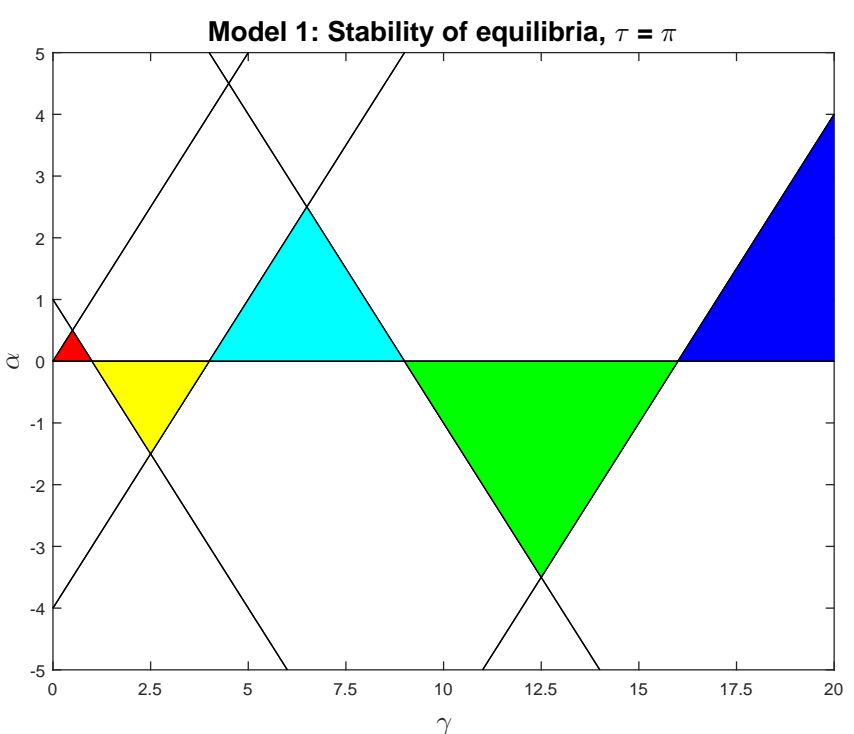

Fig. 2. Colored stability regions for the equilibrium point of system (2), with $\tau=\pi$ and arbitrary $\beta$, according to different intervals of the parameter $\gamma$. When $\tau$ has a fixed value, the Hopf curves of (2) are straight lines. The line $\alpha=\gamma$ that delimits the stability area for $0<\gamma<1$, is a static bifurcation curve and its crossing with $\bar{\alpha}_{1}$ shows a GavrilovGuckenheimer singularity.

\section{2. $\quad$ Model 2}

Now, (1) with $u=\dot{x}_{\tau}$ is taken into account where the feedback action depends on the velocity, with $\gamma, \tau>0, \alpha \neq 0$ and $\beta \in \mathbb{R}$. To avoid misunderstanding, from now on it is defined $\delta=\alpha$. Now there is a single equilibrium point: $x=0$. Equation (1) with $u=\dot{x}_{\tau}$ can also be written as

$$
\dot{x}_{1}=-\gamma x_{2}+\delta x_{1}(t-\tau)+\beta x_{1}^{2}(t-\tau), \quad \dot{x}_{2}=x_{1},
$$

with equilibrium $X_{0}=(0,0)$. For the stability analysis of the equilibrium, it must be considered the linearization of (11) about $X_{0}$, again given by

$$
\dot{c}=\bar{A} c+\bar{A}_{\tau} c(t-\tau)
$$

(see (3)), where

$$
\bar{A}=\left(\begin{array}{cc}
0 & -\gamma \\
1 & 0
\end{array}\right) \text { and } \bar{A}_{\tau}=\left(\begin{array}{cc}
\delta & 0 \\
0 & 0
\end{array}\right) .
$$

To guarantee the asymptotic stability of the equilibrium, the characteristic equation $\operatorname{det}\left(\lambda I-\bar{A}-\bar{A}_{\tau} e^{-\lambda \tau}\right)=0$, must have all its roots on the left half plane. Through the change of variables $z=\lambda \tau$ results an exponential polynomial

$$
P_{2}(z)=p\left(z, e^{z}\right)=e^{z}\left(z^{2}+\gamma \tau^{2}\right)-\delta \tau z=0 .
$$

To apply Theorem 2 but now with the hypothesis I), notice that $P_{2}$ differs from $P_{1}$ (see (4)) only about its last term. 
Remark 2.9. In Theorem 2, the condition $F^{\prime} G(y)<$ 0 means that $G(y) \neq 0$ for each root of $F$, which must be all simple (to carry out $F^{\prime}(y) \neq 0$ ).

By (13), the expression $P_{2}(i y)=F_{2}(y)+i G_{2}(y)$ leads to

$$
\begin{aligned}
& F_{2}(y)=\left(-y^{2}+\gamma \tau^{2}\right) \cos y, \\
& G_{2}(y)=\left(-y^{2}+\gamma \tau^{2}\right) \sin y-\delta \tau y .
\end{aligned}
$$

The zeros of $F_{2}$ are
a) $\bar{y}_{k}=(2 k-1) \frac{\pi}{2}, k \in \mathbb{Z}$,
b) $\breve{y}_{1,2}= \pm \sqrt{\gamma} \tau$.

Then the conditions required to the parameters to satisfy the hypothesis I) of Theorem 2 are:

a) The values of $\gamma, \tau$ and $\delta$ in (13) should not solve the equation

$$
(-1)^{k}\left(\bar{y}_{k}^{2}-\gamma \tau^{2}\right)-\delta \tau \bar{y}_{k}=0, \quad k \in \mathbb{Z} .
$$

b) As

$$
F_{2}^{\prime}(y)=-2 y \cos y-\left(-y^{2}+\gamma \tau^{2}\right) \sin y,
$$

then

$$
\gamma \tau^{2}=(2 k-1)^{2}(\pi / 2)^{2}, k \in \mathbb{Z},
$$

should not be satisfied to fulfill $F_{2}^{\prime}(y) \neq 0$, for any root $y$ of $F_{2}$.

One knows that $\tau$ verifies one of these two conditions: 1) $0<\tau<\bar{y}_{1}$ or 2) $\bar{y}_{k}<\tau<\bar{y}_{k+1}, k \in \mathbb{N}$. Notice that, again, four real parameters are involved in the stability conditions. However, the model can be reduced to a three-parameter one through the change of time scale $t^{\prime}=\sqrt{\gamma} t$, which is equivalent to just consider $\gamma=1$.

Thus, the following theorem can be set:

Theorem 4. Consider (13) and (14) with $\tau>0$, $\gamma=1$ and $\delta \neq 0$. Let be $\bar{y}_{k}, k \in \mathbb{N}$ and $\breve{y}_{1,2}$ given by (15). Assume that the parameter values do not satisfy neither (16) nor (18). All the roots of $P_{2}$ lie on the left half plane if and only if

I) For $0<\tau<\bar{y}_{1}$, it must be

$$
\delta>-\frac{\bar{y}_{1}}{\tau}+\frac{\tau}{\bar{y}_{1}}, \quad \delta<0 .
$$

II) For $\bar{y}_{k}<\tau<\bar{y}_{k+1}, k \in \mathbb{N}$, it is required:

a) If $k$ is odd, then

$\delta<-\frac{\bar{y}_{k}}{\tau}+\frac{\tau}{\bar{y}_{k}}, \quad \delta<\frac{\bar{y}_{k+1}}{\tau}-\frac{\tau}{\bar{y}_{k+1}}, \quad \delta>0$.

b) If $k$ is even, then

$\delta>\frac{\bar{y}_{k}}{\tau}-\frac{\tau}{\bar{y}_{k}}, \quad \delta>-\frac{\bar{y}_{k+1}}{\tau}+\frac{\tau}{\bar{y}_{k+1}}, \quad \delta<0$.
Proof. $\Longrightarrow$ (Necessary condition) This result is a consequence of Theorem 1. It is clear that all the zeros of $F_{2}$ given by (15) are real and effectively there are $2 k+1$ in any interval $[0,2 k \pi]$ which includes $\tau$. Now, the zeros of $G_{2}$ (see (14)) coincide with the solutions of the equation

$$
\sin y=\frac{\delta \tau y}{-y^{2}+\tau^{2}} .
$$

There must be $4 k+2$ roots in some interval $[-2 k \pi+\pi / 2,2 k \pi+\pi / 2]$ for $k$ sufficiently large, in order to assert that all the roots of $G_{2}$ are real [Bellman \& Cooke, 1963].

I) $0<\tau<\bar{y}_{1}=\pi / 2$. First observe that $G_{2}(0)=0$ (see (19) and (14)). Then, consider the interval $\left(\tau, \bar{y}_{1}\right)$. Taking into account the roots of $F_{2}(15)$, which should alternate with $G_{2}$ 's by Theorem 1 , one computes $G_{2}(\tau)=-\delta \tau^{2}, G_{2}\left(\bar{y}_{1}\right)=-\bar{y}_{1}^{2}+\tau^{2}-\delta \tau \bar{y}_{1}$ and observes that if $\delta>0$ then $G_{2}(\tau)$ and $G_{2}\left(\bar{y}_{1}\right)$ are both negative. Then it should be $\delta<0$, by Bolzano's Theorem results that $G_{2}$ has a root between $\breve{y}_{1}=\tau$ and $\bar{y}_{1}=\pi / 2$ and $G_{2}\left(\bar{y}_{1}\right)<0$. So, it follows $\delta>-\bar{y}_{1} / \tau+\tau / \bar{y}_{1}$. Now, considering again the zeros of $G_{2}$ and the interval $\left(\bar{y}_{1}, \bar{y}_{2}\right)$ it is necessary that $G_{2}\left(\bar{y}_{2}\right)=\left(-\bar{y}_{2}^{2}+\tau^{2}\right)(-1)-\delta \tau \bar{y}_{2}>0$. This means $\delta<\bar{y}_{2} / \tau-\tau / \bar{y}_{2}$, which is true under $\delta<0$. Thereby, three roots of $G_{2}$ exist in $\left[0, \bar{y}_{2}\right]$.

Now, let $0<\tau<\bar{y}_{1}<\bar{y}_{j}$. For $j$ odd, $j>$ $1, G_{2}\left(\bar{y}_{j}\right)=\left(-\bar{y}_{j}^{2}+\tau^{2}\right)-\delta \tau \bar{y}_{j}<0$, which is equivalent to $\delta>-\bar{y}_{j} / \tau+\tau / \bar{y}_{j}$, but this condition is satisfied due to $\delta>-\bar{y}_{1} / \tau+\tau / \bar{y}_{1}>-\bar{y}_{j} / \tau+\tau / \bar{y}_{j}$. For $j$ even, $G_{2}\left(\bar{y}_{j}\right)=\left(-\bar{y}_{j}^{2}+\tau^{2}\right)(-1)-\delta \tau \bar{y}_{j}>0$, which is equivalent to $\delta<\bar{y}_{j} / \tau-\tau / \bar{y}_{j}$. This assertion is valid under $\delta<0$.

So, in each interval $\left[y_{j}, y_{j+1}\right], j \geq 2, G_{2}$ has one root. Hence, there are $4 k+2$ zeros of $G_{2}$ in $[-2 k \pi+\pi / 2,2 k \pi+\pi / 2]$.

The proof for II)a) appears in Appendix B and the one for II)b) follows analogously.

$\Longleftarrow$ (Sufficient condition, sketch) In order to apply Theorem 2, it is necessary to check that $F_{2}^{\prime} G_{2}(y)<$ 0 for each $y$, an arbitrary root of $F_{2}$ (see (15)).

I) The sign of $\delta$ follows from the requirement of $F_{2}^{\prime} G_{2}\left(\breve{y}_{1,2}\right)<0$. The prime inequality results analyzing the necessary statements to fulfill $F_{2}^{\prime} G_{2}\left(\bar{y}_{1}\right)<0$. To satisfy $F_{2}^{\prime} G_{2}\left(\bar{y}_{k}\right)<0, k \geq 2$, it can be shown that the obtained conditions are sufficient, by considering separately the cases $k$ odd or even.

II) The sign of $\delta$ is established from $F_{2}^{\prime} G_{2}\left(\breve{y}_{1,2}\right)<0$, according to $k$ being odd or even. The first two inequalities in a) and b) are deduced imposing 
$F_{2}^{\prime} G_{2}\left(\bar{y}_{k}\right)<0$ and $F_{2}^{\prime} G_{2}\left(\bar{y}_{k+1}\right)<0$, when $k$ is odd or even. These conditions result sufficient to guarantee $F_{2}^{\prime} G_{2}\left(\bar{y}_{i}\right)<0$, where $i<k$ or $i>k+1$. To show this last it is necessary to consider the four different cases which result for $i$ odd or even.

The details to prove the condition II)a) appear in Appendix B. The other cases are similar.

It still remains to test that $F_{2}^{\prime} G_{2}\left(\bar{y}_{k}\right)<0$ for each $\bar{y}_{k}<0$. But, taking into account that $F_{2}^{\prime} G_{2}$ is even, then the whole proof results complete now.

Corollary 2.2. The equilibrium of system (11) results asymptotically stable under the necessary and sufficient conditions established in Theorem 4.

Remark 2.10. A different proof of Theorem 4 has also been obtained through frequency-domain methods using the Nyquist stability criterion.

Remark 2.11. The whole stability analysis for the trivial equilibrium in an equation like (1) with $u=$ $\dot{x}_{\tau}$, which includes a term in $\dot{x}$ in its left member can be found in Bellman and Cooke [1963].

Remark 2.12. When (16) is satisfied, the roots of (13) are on the imaginary axis. Then, the Hopf surfaces in the three dimensional parameter space $\gamma-\tau-\delta$ (for system (11)), or alternatively, fixing the parameter $\gamma$, the Hopf curves $\delta_{k}$ in the $\tau-\delta$ plane are determined by

$$
\delta=\delta_{k}(\tau)=(-1)^{k}\left(\frac{\bar{y}_{k}}{\tau}-\frac{\gamma \tau}{\bar{y}_{k}}\right) .
$$

Figure 3 shows a few stability areas, some Hopf curves $\delta_{k}$ (see (20)) and multiple resonant points in the $\tau-\delta$ plane, with $\gamma=1$. The Hopf curves $\delta_{k}$ intersect the $\tau$ axis at the points $\tau=\bar{y}_{k}$. The curves $\delta_{k}$ and $\delta_{j}$ intersect each other only if $k$ and $j$ have different parity, at the points with coordinates

$$
(\tau, \delta)=\left(\left(\bar{y}_{k} \bar{y}_{j}\right)^{1 / 2},(-1)^{k}\left(\bar{y}_{k}-\bar{y}_{j}\right)\left(\bar{y}_{k} \bar{y}_{j}\right)^{-1 / 2}\right),
$$

which are $2 k-1: 2 j-1$ resonant points $(k<j)$. At the resonance points, (13) has two pair of pure imaginary solutions, namely $\pm i \bar{y}_{k} / \tau, \pm i \bar{y}_{j} / \tau$ satisfying $\bar{y}_{k} / \bar{y}_{j}=(2 k-1) /(2 j-1)$. This situation is related in system (11) with the interaction between two limit cycles, whose frequencies are $(2 k-1) \pi /(2 \tau)$ and $(2 j-1) \pi /(2 \tau)$ approximately. Thereby, the intersection between the Hopf curves $\delta_{1}$ and $\delta_{2}$ results in a $1: 3$ resonance point.

Remark 2.13. An equivalent result can be established by the assignment of a fixed value of $\tau$ in (13) and (15). In this case, the resulting restrictions between $\gamma$ and $\delta$ are linear. Related with this assumption, the complete stability analysis of a general delay differential equation which includes (12) can be found in Stépán [1989].

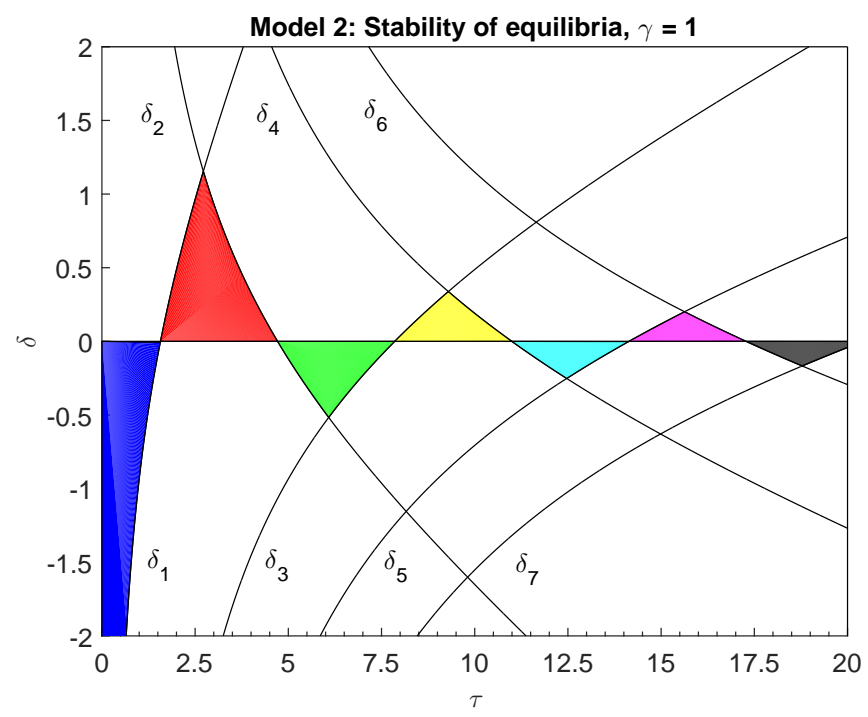

Fig. 3. Stability regions for the equilibrium point of (11) with $\gamma=1$ and arbitrary $\beta$. According to Theorem 4, regions in red, yellow and magenta correspond to $k=1,3,5$, respectively, of situation (II)a), while areas in green, cyan and black refer to $k=2,4,6$, under situation (II)b). Finally the blue domain represents the situation I).

\section{Cycles Stability Analysis: A Frequency-Domain Approach}

The stability results for the equilibrium can be combined with the computation of the curvature coefficient provided by the frequency-domain method [Mees \& Chua, 1979; Mees, 1981; Moiola \& Chen, 1996] to know the cycles stability.

\subsection{Model 1}

It is proposed a feedback representation of the system (2) like

$$
\left\{\begin{aligned}
\dot{X} & =A X+B h(y(t-\tau)), \\
h(y) & =-\alpha y+\beta y^{2}, \quad y=-C X,
\end{aligned}\right.
$$

where

$$
A=\left(\begin{array}{cc}
0 & -\gamma \\
1 & 0
\end{array}\right), B=\left(\begin{array}{l}
1 \\
0
\end{array}\right), C=B^{t}
$$


In the frequency domain the equilibrium can be found through

$$
y=-G(0) h(y),
$$

where $G$ is the transfer matrix for the linear part defined as

$$
G(s)=C(s I-A)^{-1} B e^{-s \tau}=\frac{e^{-s \tau}}{\gamma+s^{2}} .
$$

The solutions of (21) are $y=0$ and $y=(\alpha-\gamma) \beta^{-1}$. Then, it will be considered only the analysis related with $y=0$, as in Section 2.1. The expression of the characteristic function, which equals the (linearized) loop gain, is

$$
\lambda(s)=G(s) J=-\frac{\alpha e^{-s \tau}}{\gamma+s^{2}}, \text { where } J=h^{\prime}(0)=-\alpha .
$$

The appearance of a Hopf bifurcation related with the trivial equilibrium can be shown by solving $\lambda(i \omega)=-1, \omega \in \mathbb{R}^{+}$[Moiola \& Chen, 1996]. Considering this condition particularly with (23) and splitting into real and imaginary parts one gets

$$
\alpha \cos \omega \tau=\gamma-\omega^{2}, \quad \alpha \sin \omega \tau=0 .
$$

As $\alpha \neq 0$, it must be $\omega \tau=y_{k}=k \pi, k \in \mathbb{N}$, and then the expressions for the Hopf curves in the $\tau-\alpha$ plane are:

$$
\alpha=\alpha_{k}(\tau)=(-1)^{k}\left(-y_{k}^{2} / \tau^{2}+\gamma\right),
$$

agreeing with (10).

Under the frequency-domain approach, the curvature coefficient of a cycle that arises from a Hopf bifurcation can be computed through

$$
\sigma(\omega)=-\operatorname{Re}\left(\frac{G(i \omega) p(i \omega)}{G^{\prime}(i \omega) J}\right),
$$

where according with Mees and Chua [1979], a simplified expression for $p(i \omega)$ for SISO systems is

$$
p(i \omega)=D_{2}\left(\frac{1}{2} V_{22}+V_{02}\right)+D_{3},
$$

but $D_{2}=h^{\prime \prime}(0)=2 \beta$ and $D_{3}=h^{\prime \prime \prime}(0)=0$, so only $V_{02}$ and $V_{22}$ are pending. In engineering terms, $V_{02}$ is related with the bias for the correction of the equilibrium point due to the nonlinearities; $V_{22}$ is connected to the amplitude of the second harmonic of the oscillatory solution and $p(i \omega)$ is associated to the amplitude of the term of fundamental frequency. The closed-loop transfer function is

$$
H(s)=(1+G(s) J)^{-1} G(s),
$$

thus defining $\rho=\gamma-\alpha$ (which is nonzero by the assumption that $\gamma \neq \alpha$ ) one has $H(0)=\rho^{-1}$, $H(i 2 \omega)=\left(\rho-4 \omega^{2}\right)^{-1}$ and finally

$$
\begin{aligned}
& V_{02}=-\frac{1}{4} H(0) D_{2}=-\frac{1}{2} \beta \rho^{-1}, \\
& V_{22}=-\frac{1}{4} H(i 2 \omega) D_{2}=-\frac{1}{2} \beta\left(\rho-4 \omega^{2}\right)^{-1} .
\end{aligned}
$$

Then, according with (26),

$$
p(i \omega)=-\frac{\beta^{2}}{2}\left(\frac{2}{\rho}+\frac{1}{\rho-4 \omega^{2}}\right) .
$$

Using (22), (28) and fixing $\omega_{k}=y_{k} / \tau=k \pi / \tau$, $k \in \mathbb{N}$, one has

$$
G\left(i \omega_{k}\right) p\left(i \omega_{k}\right)=-\frac{\beta^{2}(-1)^{k} \tau^{2}\left(3 \rho \tau^{2}-8 y_{k}^{2}\right)}{2 \rho\left(\gamma \tau^{2}-y_{k}^{2}\right)\left(\rho \tau^{2}-4 y_{k}^{2}\right)} .
$$

Besides, as

$$
\begin{aligned}
G^{\prime}\left(i \omega_{k}\right) J & =\frac{\alpha(-1)^{k+1} \tau\left[\left(\gamma \tau^{2}-y_{k}^{2}\right)+2 i y_{k}\right]}{\left(\gamma \tau^{2}-y_{k}^{2}\right)^{2}} \\
& =\tau+\frac{2 i \omega(-1)^{k+1}}{\alpha \tau^{2}},
\end{aligned}
$$

finally using the short notation $\sigma(k)=\sigma\left(i \omega_{k}\right)$, Eq. (25) results

$$
\sigma(k)=\frac{\beta^{2}(-1)^{k} \tau^{3}\left(3 \rho \tau^{2}-8 y_{k}^{2}\right)}{2 \rho\left(\gamma \tau^{2}-y_{k}^{2}\right)\left(\rho \tau^{2}-4 y_{k}^{2}\right)} .
$$

Notation: Hereinafter the subscript $e$ or $o$ refers to $k$ even or odd, respectively.

The sign of (29) gains sense when it is evaluated on points of a Hopf curve. For a Hopf curve with $k$ even (see (24)), one has $\alpha=\alpha_{k}(\tau)=-y_{k}^{2} / \tau^{2}+\gamma$, i.e. $\rho=y_{k}^{2} / \tau^{2}$. Replacing into (29) results $\sigma_{e}(k)=$ $5 \beta^{2} \tau^{3} /\left(6 \alpha y_{k}^{2}\right)$ then $\operatorname{sgn}\left(\sigma_{e}(k)\right)=\operatorname{sgn}(\alpha)$. Moreover if $k$ is odd, as $\alpha=\alpha_{k}=y_{k}^{2} / \tau^{2}-\gamma$, i.e. $\rho=$ $2 \gamma-y_{k}^{2} / \tau^{2}$, one gets

$$
\sigma_{o}(k)=-\frac{\beta^{2} \tau\left(5 y_{k}^{2} / \tau^{2}+6 \alpha\right)}{2 \alpha\left(-y_{k}^{2} / \tau^{2}+2 \alpha\right)\left(3 y_{k}^{2} / \tau^{2}+2 \alpha\right)},
$$

but the study of the sign of $\sigma_{o}(k)$ requires a bit more work. Defining four auxiliary curves in the $\tau-\alpha$ plane, i.e., $C_{1}: \alpha=y_{k}^{2} /\left(2 \tau^{2}\right), C_{2}: \alpha=0$, $C_{3}: \alpha=-5 y_{k}^{2} /\left(6 \tau^{2}\right)$ and $C_{4}: \alpha=-3 y_{k}^{2} /\left(2 \tau^{2}\right)$ results

$$
\begin{aligned}
& \operatorname{sgn}\left(\sigma_{o}(k)\right)= \\
& \left\{\begin{array}{ccl}
-1, & y_{k}^{2} /\left(2 \tau^{2}\right)<\alpha & \Leftrightarrow 0<\tau<\tau_{1}, \\
1, & 0<\alpha<y_{k}^{2} /\left(2 \tau^{2}\right) & \Leftrightarrow \tau_{1}<\tau<\tau_{2}, \\
-1, & -5 y_{k}^{2} /\left(6 \tau^{2}\right)<\alpha<0 & \Leftrightarrow \tau_{2}<\tau<\tau_{3}, \\
1,-3 y_{k}^{2} /\left(2 \tau^{2}\right)<\alpha<-5 y_{k}^{2} /\left(6 \tau^{2}\right) & \Leftrightarrow \tau_{3}<\tau<\tau_{4}, \\
-1, & \alpha<-3 y_{k}^{2} /\left(2 \tau^{2}\right) & \Leftrightarrow \tau>\tau_{4},
\end{array}\right.
\end{aligned}
$$

where $\tau_{i}$ is defined as the intersection between the Hopf curve $\alpha=\alpha_{k}=y_{k}^{2} / \tau^{2}-\gamma, k$ odd, and 
$C_{i}, 1 \leq i \leq 4$. So, as the points $\tau_{i}$ depend on the number $k$, they will be noted as

$$
\begin{gathered}
\tau_{1(k)}=k \pi / \sqrt{2 \gamma}, \quad \tau_{2(k)}=k \pi / \sqrt{\gamma}, \\
\tau_{3(k)}=k \pi \sqrt{11} / \sqrt{6 \gamma}, \tau_{4(k)}=k \pi \sqrt{5} / \sqrt{2 \gamma} .
\end{gathered}
$$

Thus, there are three points in the curve $\alpha_{k}, k$ odd, where $\sigma_{o}$ is indefinite corresponding to $\tau_{1(k)}, \tau_{2(k)}$ and $\tau_{4(k)}$. The first one corresponds to a GavrilovGuckenheimer (zero-Hopf) singularity. The point which has $\tau$ - coordinate $\tau_{3(k)}$, where the coefficient $\sigma_{o}$ vanishes, gives place to the appearance of a fold of cycles bifurcation curve. Figure 4 shows two branches of periodic solutions close to $R=$ $\left(\tau_{3(1)}, \alpha_{1}\left(\tau_{3(1)}\right)\right)=(4.2536,-0.4545)$, one which exhibits the fold of cycles and the other corresponds to the classic Hopf branch. These continuations have been obtained using the software DDE-Biftool [Engelborghs et al., 2002]. The intersection between two curves $\alpha_{k}$ and $\alpha_{2 k}$ with $k$ odd is a 1:2 resonance point and its $\tau$ - coordinate is $\tau_{4(k)}$, so $\sigma_{o}$ is undetermined there.
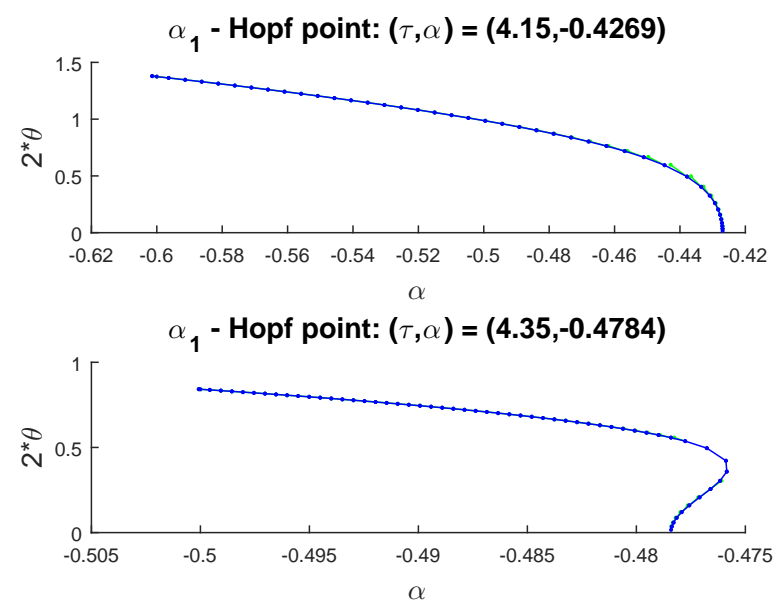

Fig. 4. Branches of periodic solutions of system (2), with $\gamma=1$ and arbitrary $\beta$, born at $\alpha_{1}$ - Hopf points, close to $R=(\tau, \alpha)=(4.2536,-0.4545)$. (Above: Stable branch. Below: Unstable branch with fold of limit cycles). Here, $\theta$ represents an approximation of the amplitude of the cycle.

Remark 3.1. The outcomes achieved in this section for system (2) with $\gamma=1$, can also be generalized for an arbitrary positive value of $\gamma$.

According with the analysis of the sign of the curvature coefficient for any Hopf curve $\alpha_{k}$ and from the stability regions shown in Fig. 1, a synthesis of the stability outcomes on the Hopf curves with $\gamma=1$ can be observed in Fig. 5 .

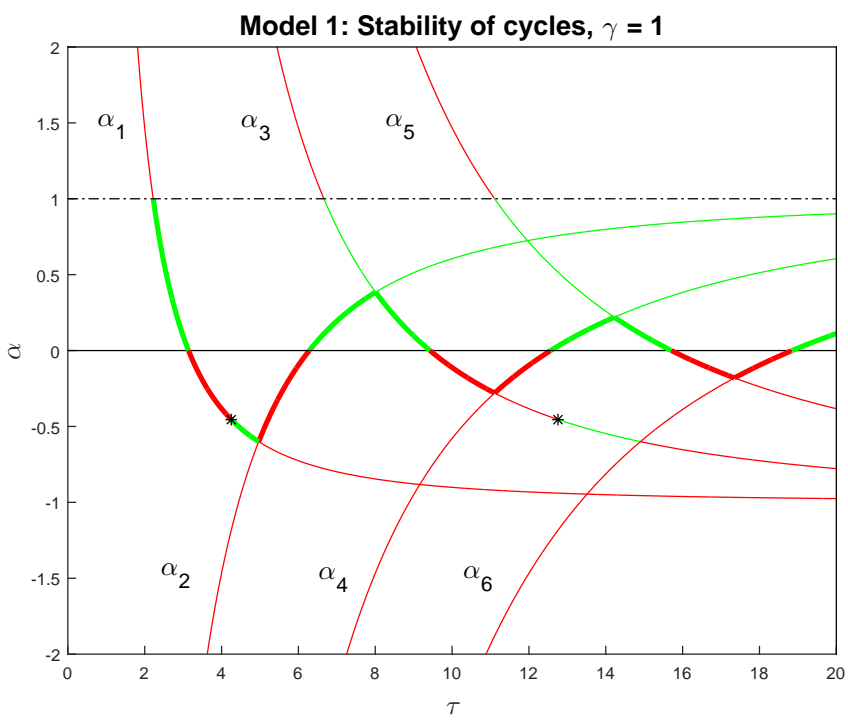

Fig. 5. Some Hopf curves (24) of system (2) with $\gamma=1$ and arbitrary $\beta$, where red and green denote negative and positive values of $\sigma(k)$, respectively. Colored thick lines show stability of emergent limit cycles (red - stable, green - unstable), due to a change of stability of the equilibrium point (see Fig. 1). The asterisks correspond to the located points over the curves $\alpha_{1}$ and $\alpha_{3}$ where $\sigma$ vanishes.

\subsection{Model 2}

System (11) can be written as

$$
\left\{\begin{aligned}
\dot{X} & =A X+B h(y(t-\tau)), \\
h(y) & =-\delta y+\beta y^{2}, \quad y=-C X,
\end{aligned}\right.
$$

where

$$
A=\left(\begin{array}{cc}
0-\gamma \\
1 & 0
\end{array}\right), B=\left(\begin{array}{l}
1 \\
0
\end{array}\right), C=B^{t} .
$$

Thus, the transfer function for the linear part becomes

$$
G(s)=C(s I-A)^{-1} B e^{-s \tau}=\frac{s e^{-s \tau}}{\gamma+s^{2}} .
$$

The equilibrium $y=0$ comes from solving the equation $-y=G(0) h(y)$. In this case, the characteristic eigenvalue is

$$
\lambda(s)=G(s) J=-\frac{\delta s e^{-s \tau}}{\gamma+s^{2}}, \text { where } J=h^{\prime}(0)=-\delta .
$$

The critical condition at the Hopf bifurcation point, i.e., $\lambda(i \omega)=-1$, gives this system of equations

$$
\omega^{2}-\gamma+\delta \omega \sin \omega \tau=0, \quad \delta \omega \cos \omega \tau=0 .
$$

Then, as $\omega>0$ and $\delta \neq 0$, it should be $\omega \tau=$ $(2 k-1) \pi / 2=y_{k}, k \in \mathbb{N}$ as well as

$$
\omega^{2}-\gamma-\delta \omega(-1)^{k}=0,
$$


which resumes the Hopf bifurcation condition and results equivalent to (20) for $k \in \mathbb{N}$ after replacing $\omega_{k}=y_{k} / \tau$.

From (30) follows $D_{2}=h^{\prime \prime}(0)=2 \beta$ and $D_{3}=$ $h^{\prime \prime \prime}(0)=0$. From (27) one has

$$
H(0)=0, \quad H(i 2 \omega)=-\frac{i 2 \omega}{\left(\gamma-4 \omega^{2}\right)+\delta i 2 \omega},
$$

and finally

$$
\begin{aligned}
& V_{02}=-\frac{1}{4} H(0) D_{2}=0, \\
& V_{22}=-\frac{1}{4} H(i 2 \omega) D_{2}=\frac{i \eta \omega}{\left(\gamma-4 \omega^{2}\right)+\delta i 2 \omega} .
\end{aligned}
$$

Provided that $p(i \omega)=\eta V_{22}$, now (see (25)) it is defined

$$
a u x=G(i \omega) p(i \omega)=-\frac{\beta^{2} \omega^{2} e^{-i \omega \tau}}{\left(\gamma-\omega^{2}\right)\left(\gamma-4 \omega^{2}+\delta i 2 \omega\right)} .
$$

Notation: In what follows, the expression aux $(k)$ will mean $\operatorname{aux}\left(i \omega_{k}\right)$ where $\omega_{k} \tau=(2 k-1) \pi / 2$, e.g. $\operatorname{aux}(1)$ denotes $\operatorname{aux}\left(i \omega_{1}\right)$ where $\omega_{1} \tau=\pi / 2$. The same notation is extended for $G^{\prime}$ and $\sigma$ as for the previous case. Again, the subscript $o$ or $e$ means $k$ odd or even, respectively.

To get the expression of $\sigma$, two possibilities must be considered: $\omega_{k} \tau=(2 k-1) \pi / 2$, where $k$ is odd or even. Then, the whole analysis gives place to three different situations that will be developed below and thus the stability analysis will be completed. Now, the sign of the curvature coefficient will be determined for the two cases mentioned in Theorem 4. Case I) First using (32), again with $\gamma=1$, for an arbitrary odd value of $k$, Eqn. (33) results

$$
\operatorname{aux}_{o}(k)=\frac{\beta^{2} i}{\delta\left(-3 \omega_{k}+\delta(1+2 i)\right)},
$$

then if $k=1$ one has

$$
\operatorname{aux}_{o}(1)=\frac{2 \tau \beta^{2} i}{\delta(-3 \pi+2 \tau \delta(1+2 i))} .
$$

From (31), it follows that

$$
G_{o}^{\prime}(1)=\left.G^{\prime}(s)\right|_{s=i \omega_{1}}=-i \frac{(-2-i \pi) \delta \pi \tau+8 \tau^{2}}{\delta^{2} \pi^{2}} .
$$

For $\omega_{1} \tau=\pi / 2$, i.e. $k=1$, and over the Hopf curve $\delta_{1},(25)$ becomes

$$
\sigma_{o}(1)=-\operatorname{Re}\left(\frac{a u x_{o}(1)}{-\delta G_{o}^{\prime}(1)}\right),
$$

and after some tedious calculations, it leads to $\operatorname{sgn}\left(\sigma_{o}(1)\right)=-\operatorname{sgn}\left(P_{1}\right)$, where

$$
P_{1}=2 \pi \tau(\pi-1) \delta^{2}+\left(8 \tau^{2}+3 \pi^{2}\right) \delta-12 \pi \tau \text {. }
$$

Moreover, to find where $\sigma(1)=0, P_{1}=0$ under $\delta=\delta_{1}(\tau)=-\pi /(2 \tau)+2 \tau / \pi$ must be solved. So the unique point of $\delta_{1}$ where $\sigma=0$ results $Q=(\tau, \delta)=(2.3979,0.8715)$ where the nearby dynamics are similar to those found near point $R$, in the previous subsection. If $\tau<2.3979$, due to $\sigma>0$ the emergent cycles are unstable. Close to $Q$, branches of periodic solutions exhibit the cycle folds, where two limit cycles collide and disappear. On the contrary, for $2.3979<\tau<\sqrt{y_{1} y_{2}}=\sqrt{3} \pi / 2$, as $\sigma<0$ the cycles result stable (classic Hopf branch). Otherwise, for $\tau>\sqrt{3} \pi / 2$, the emergent orbits are unstable. This situation is identical to the one described in Model 1, close to the point $R$, and illustrated in Fig. 4.

Besides, if one replaces $\delta \tau=-\pi / 2+2 \tau^{2} / \pi$ into $\tau P_{1}$ (see (34)) and then multiplies by 2 , it is obtained another expression $\tilde{P}_{1}$ in the variable $\tau$ that can be written as

$\tilde{P}_{1}(\tau)=16\left(1+\pi^{-1}\right) \tau^{4}-4 \pi(2 \pi+3) \tau^{2}+\pi^{3}(\pi-4)$.

Then, applying Descartes' rule it can be shown that $\tilde{P}_{1}$ has exactly one positive root, as commented before.

Case II)a) Now, it is analyzed the case with $k$ odd but $k \geq 3$, remaking the steps done before for $\omega_{k} \tau=(2 k-1) \pi / 2=a \pi / 2$, where $a=2 k-1$ $\geq 5$ if $k \geq 3$. Furthermore, due to (32), one has $\omega^{2}-1+\delta \omega=0$ and then

$$
\operatorname{aux}_{o}(k)=\frac{2 \tau \beta^{2} i}{\delta(-3 a \pi+2 \tau \delta(1+2 i))},
$$

as well as

$$
G_{o}^{\prime}(k)=-i \frac{(-2-i(2 k-1) \pi) \delta(2 k-1) \pi \tau+8 \tau^{2}}{\delta^{2}(2 k-1)^{2} \pi^{2}} .
$$

Then for an arbitrary odd $k$ with $k \geq 3$ from

$$
\sigma_{o}(k)=-\operatorname{Re}\left(\frac{a u x_{o}(k)}{-\delta G_{o}^{\prime}(k)}\right),
$$

follows $\operatorname{sgn}\left(\sigma_{o}(k)\right)=-\operatorname{sgn}\left(P_{k}\right)$, where

$$
P_{k}=[2 \pi \tau a(\pi a-1)] \delta^{2}+\left(8 \tau^{2}+3 \pi^{2} a^{2}\right) \delta-12 \pi \tau a \text {. }
$$

Once again, to find the point along the Hopf curve where $\sigma=0$, it must be solved $P_{k}=0$ and $\delta=\delta_{k}(\tau)=-a \pi /(2 \tau)+2 \tau /(a \pi)$. To locate the intersections one can proceed as in the case with $k=1$. Nevertheless, if $k$ is odd with $k \geq 3$ there are two solutions. This result also comes out substituting $\tau \delta=-a \pi / 2+2 \tau^{2} /(a \pi)$ into $\tau P_{k}$ (see (35)) and then multiplying by 2 . Thus, it is attained $P_{(o) k}$ which has the general form:

$$
P_{(o) k}(\tau)=R_{o} \tau^{4}+S_{o} \tau^{2}+T_{o}
$$


where $R_{o}=16\left[1+(\pi a)^{-1}\right], S_{o}=-4 \pi a(2 \pi \alpha+3)$ and $T_{o}=\pi^{3} a^{3}(\pi a-4)$. Thus $P_{(o) k}=0$ can be solved analytically yielding

$$
\tau^{2}=\frac{\pi^{2} a^{2}(2 \pi a+3 \pm \sqrt{25+24 \pi a})}{8(\pi a+1)} .
$$

As $a \geq 5$, then $\tau^{2}$ takes two different positive values. So (36) has two positive roots and (35) becomes zero for two different $(\tau, \delta)$ pairs. For instance, when $k=3$, the roots are $\tau_{1}=5.1497, \tau_{2}=10.0271$ and the corresponding $\delta$ values are -0.869453 and 0.4934145 . Thus, considering the curve $\delta_{3}$ and the regions of stability of the equilibrium point (see Fig. 3), the emergent orbits result stable only for $\sqrt{y_{2} y_{3}}=\sqrt{15} \pi / 2<\tau<\sqrt{35} \pi / 2=\sqrt{y_{3} y_{4}}$. This outcome can be generalized for any Hopf curve $\delta_{k}$, with $k$ odd, $k \geq 3$, for the interval $I=$ $\left(\sqrt{y_{k-1} y_{k}}, \sqrt{y_{k} y_{k+1}}\right)$.

In summary, it has been demonstrated that $\delta_{1}$ has a unique point where the curvature coefficient vanishes whereas $\delta_{k}$ has exactly two points where $\sigma=0$ if $k$ is odd and $k \geq 3$. This result is original in system (11) and appears after considering the variation of several parameters.

Case II)b) Finally if $k$ is even, taking into account that $\omega_{k} \tau=(2 k-1) \pi / 2=a \pi / 2$, where $a \geq 3$, due to (32) and (33) results

$$
\operatorname{aux}_{e}(k)=\frac{2 \tau \beta^{2} i}{\delta(-3 a \pi+2 \tau \delta(-1+2 i))}
$$

and

$$
G_{e}^{\prime}(k)=i \frac{(2+i a \pi) \delta a \pi \tau+8 \tau^{2}}{\delta^{2} a^{2} \pi^{2}} .
$$

In general, for even $k$, as

$$
\sigma_{e}(k)=-\operatorname{Re}\left(\frac{a u x_{e}(k)}{-\delta G_{e}^{\prime}(k)}\right),
$$

then $\operatorname{sgn}\left(\sigma_{e}(k)\right)=-\operatorname{sgn}\left(P_{k}\right)$, where

$$
P_{k}=[2 \pi \tau a(\pi a+1)] \delta^{2}+\left(8 \tau^{2}+3 \pi^{2} a^{2}\right) \delta+12 \pi \tau a .
$$

Making the substitution $\tau \delta=a \pi / 2-2 \tau^{2} /(a \pi)$ into $\tau P_{k}$ above, follows

$$
P_{(e) k}(\tau)=R_{e} \tau^{4}+S_{e} \tau^{2}+T_{e}=0,
$$

where $R_{e}=16\left[1-(\pi a)^{-1}\right], S_{e}=-4 \pi a(2 \pi a-3)$ and $T_{e}=\pi^{3} a^{3}(\pi a+4)$. Thus, solving (37) it is easy to find that the values of $\tau^{2}$ result complex because $a \geq 3$. Then (37) is always nonzero since its roots are not real and definitely $P_{k}$ is always nonzero.

Thereby and in brief, it has been proved that if $k$ is even, then $\sigma$ is always negative along the
Hopf curve $\delta_{k}$. Taking into account Fig. 3, the emergent orbits are stable specifically if $\tau \in I=$ $\left(\sqrt{y_{k-1} y_{k}}, \sqrt{y_{k} y_{k+1}}\right)$.

In agreement with all the computations of this section for system (11), as well as the outcomes of Section 2.2, reflected in Fig. 3, some representative results about stability over a few Hopf curves are shown in Fig. 6.

Remark 3.2. The deductions achieved in this section for system (11) with $\gamma=1$, can also be generalized for an arbitrary positive value of $\gamma$.

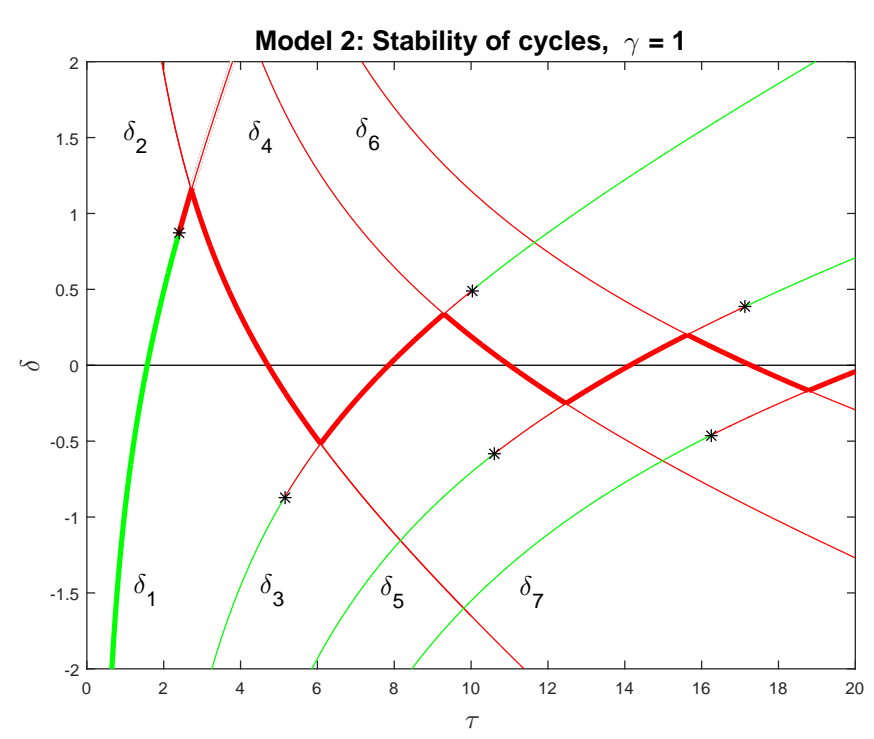

Fig. 6. Some Hopf curves (24) of system (11) with $\gamma=1$ and arbitrary $\beta$, where red and green denote negative and positive values of $\sigma$, respectively. Colored thick lines show stability of emergent limit cycles (red - stable, green - unstable), due to a change of stability of the equilibrium point (see Fig. 3). The asterisks correspond to points over the curves $\delta_{1}$, $\delta_{3}, \delta_{5}$ and $\delta_{7}$ (one in $\delta_{7}$ is not shown) where $\sigma$ vanishes.

\section{Conclusions}

Generalizations of previous analyzed models, now including more parameters, have been considered to gain clarity and perspective about the whole dynamics. The results about stability and bifurcations of equilibria have been overtaken through the theory of exponential polynomials and the location of their roots. The frequency-domain methodology has been applied to study Hopf bifurcation phenomena, achieving some interesting cycles stability outcomes and locating particular degeneracies. 


\section{Acknowledgments}

This work was supported by Universidad Nacional de Río Negro (PI 40/A623), Universidad Nacional del Sur (PGI 24/K064), ANPCyT (PICT 20142161) and CONICET (PIP 112-201201-00144).

\section{Appendix A}

Proof. [Theorem 3] $\Longrightarrow$ (Necessary condition)

Case IIa): Suppose $y_{k}<\tau<y_{k+1}, k$ odd. By reduction to the absurd, consider that $\alpha>0$. According with Theorem 1 and using Bolzano's theorem, as the roots of $F_{1}$ and $G_{1}(6)$ should alternate and $F_{1}(\tau)=-\alpha \tau^{2}<0$, then $F_{1}\left(y_{k}\right)=y_{k}^{2}-\tau^{2}-\alpha \tau^{2}>$ 0 and $F_{1}\left(y_{k+1}\right)=-y_{k+1}^{2}+\tau^{2}-\alpha \tau^{2}>0$. But $F_{1}\left(y_{k}\right)>0$ implies that $\alpha<y_{k}^{2} / \tau^{2}-1<0$ and $F_{1}\left(y_{k+1}\right)>0$ yields $\alpha<-y_{k+1}^{2} / \tau^{2}+1<0$, so $\alpha$ can not be positive. So $\alpha<0, \alpha>y_{k}^{2} / \tau^{2}-1$ and also $\alpha>-y_{k+1}^{2} / \tau^{2}+1$. Then $F_{1}$ has two roots in $\left(y_{k}, y_{k+1}\right)=(k \pi,(k+1) \pi)$.

Besides $F_{1}(0)=(1-\alpha) \tau^{2}>0$. Now, consider $y_{i}<y_{k}$. For $i<k$, odd, $F_{1}\left(y_{i}\right)=y_{i}^{2}-\tau^{2}-\alpha \tau^{2}<0$, which is equivalent to $\alpha>y_{i}^{2} / \tau^{2}-1$. This result is true under $\alpha>y_{k}^{2} / \tau^{2}-1$. For $i<k$, even, $F_{1}\left(y_{i}\right)=\left(-y_{i}^{2}+\tau^{2}\right)-\alpha \tau^{2}>0$, leads to $\alpha<-y_{i}^{2} / \tau^{2}+1$. This assertion is valid under $\alpha<0$ due to $0<-y_{i}^{2} / \tau^{2}+1$ if $y_{i}<y_{k}<\tau<y_{k+1}$. Analogously it can be proved that $F\left(y_{j}\right) F\left(y_{j+1}\right)<0$ for each $j \geq k+1$. Thereby, one has $2 k+1$ roots of $F_{1}$ in $[0,2 k \pi]$. So, all the roots of $F_{1}$ result real.

$\Longleftarrow$ (Sufficient condition)

Case $\mathbf{I}$ ): In order to apply Theorem 2, it is necessary to set conditions to satisfy $F_{1} G_{1}^{\prime}(y)>0$ for any root $y$ of $G_{1}$ given by (6) with $\gamma=1$. To prove this statement, the work is divided in two parts considering 1) $\left.\hat{y}_{1,2}= \pm \tau ; 2\right) y_{k}=k \pi, k \in \mathbb{N}^{0}$.

Let $0=y_{0}<\tau<y_{1}=\pi$.

1) Analysis with $\hat{y}_{1,2}$.

Through (5) and (8) one can establish $F_{1} G_{1}^{\prime}>0$ for $\hat{y}_{1,2}= \pm \tau$ where

$$
G_{1}^{\prime}\left(\hat{y}_{1,2}\right)=-2 \tau \sin \tau, \quad F_{1}\left(\hat{y}_{1,2}\right)=-\alpha \tau^{2} .
$$

As $0=y_{0}<\tau<y_{1}=\pi, \sin \tau>0$ and by the requirement $F_{1} G_{1}^{\prime}\left(\hat{y}_{1,2}\right)=2 \alpha \tau^{3} \sin \tau>0$, follows $\alpha>0$.

2) Analysis with $y_{k}$.

Through (5) and (8), evaluating for $y_{0}=0$, yields $G_{1}^{\prime}\left(y_{0}\right)=\tau^{2}$ and $F_{1}\left(y_{0}\right)=(1-\alpha) \tau^{2}$. As $F_{1} G_{1}^{\prime}\left(y_{0}\right)>$ 0 is necessary, results the condition $\alpha<1$.

Now, considering the root $y_{1}$ one has $G_{1}^{\prime}\left(y_{1}\right)=$ $-\left(-y_{1}^{2}+\tau^{2}\right), \quad F_{1}\left(y_{1}\right)=-\left(-y_{1}^{2}+\tau^{2}\right)-\alpha \tau^{2}$. Due to $0<\tau<y_{1}$, it yields $G_{1}^{\prime}\left(y_{1}\right)=y_{1}^{2}-\tau^{2}>0$, then to fulfill $F_{1} G_{1}^{\prime}\left(y_{1}\right)>0$ it must be

$F_{1}\left(y_{1}\right)=-\left(-y_{1}^{2}+\tau^{2}\right)-\alpha \tau^{2}>0 \Leftrightarrow \alpha<y_{1}^{2} / \tau^{2}-1$.

It is still pending to prove that $F_{1} G_{1}^{\prime}\left(y_{k}\right)>0$ for any $y_{k}$, with $k \geq 2$ and $0<\tau<y_{1}<y_{k}$. Two options must be considered $: k$ odd or $k$ even.

- $k$ odd, $k \geq 3$ : By $(8) G_{1}^{\prime}\left(y_{k}\right)=y_{k}^{2}-\tau^{2}>0$, to fulfill $F_{1} G_{1}^{\prime}\left(y_{k}\right)>0$ it must be $F_{1}\left(y_{k}\right)=-\left(-y_{k}^{2}+\tau^{2}\right)-$ $\alpha \tau^{2}>0 \Leftrightarrow \alpha<y_{k}^{2} / \tau^{2}-1$, but this condition is satisfied under (A.1) due to $y_{1}^{2} / \tau^{2}-1<y_{k}^{2} / \tau^{2}-1$.

- $k$ even, $k \geq 2$ : Due to (8) $G_{1}^{\prime}\left(y_{k}\right)=-y_{k}^{2}+\tau^{2}<0$, to supply $F_{1} G_{1}^{\prime}\left(y_{k}\right)>0$ one needs $\alpha>-y_{k}^{2} / \tau^{2}+1$. The last inequality is satisfied if $\alpha>0$ due to $0<\tau<y_{1}<y_{k}$ and $0>-y_{k}^{2} / \tau^{2}+1$.

Case IIa): As the previous case, according to Theorem 2 , it is necessary to set conditions to satisfy $F_{1} G_{1}^{\prime}(y)>0$ for any root $y$ of $G_{1}$ given by (6). Again, the work is divided in two parts considering 1) $\left.\hat{y}_{1,2}= \pm \tau ; 2\right) y_{k}=k \pi, k \in \mathbb{N}^{0}$.

Now, let $0<y_{k}<\tau<y_{k+1}, y_{k}=k \pi, y_{k+1}=$ $(k+1) \pi$ and $\gamma=1$, where $k$ is odd.

1) Analysis with $\hat{y}_{1,2}= \pm \tau$.

Through (5) and (8), $F_{1}\left(\hat{y}_{1,2}\right)=-\alpha \tau^{2}, G_{1}^{\prime}\left(\hat{y}_{1,2}\right)=$ $-2 \tau \sin \tau$ and by the stability condition $F_{1} G_{1}^{\prime}(y)>$ 0 for the zeros of $G_{1}$, becomes $F_{1} G_{1}^{\prime}\left(\hat{y}_{1,2}\right)=$ $2 \alpha \tau^{3} \sin \tau>0$. As $y_{k}<\tau<y_{k+1}, y_{k}=k \pi, k$ odd, results $\sin \tau<0$ then it must be $\alpha<0$.

2) Analysis with $y_{k}$.

Through (5) and (8) evaluating for $y_{k}$ one has

$$
\begin{aligned}
G_{1}^{\prime}\left(y_{k}\right) & =(-1)^{k}\left(-y_{k}^{2}+\tau^{2}\right), \\
F_{1}\left(y_{k}\right) & =(-1)^{k}\left(-y_{k}^{2}+\tau^{2}\right)-\alpha \tau^{2} .
\end{aligned}
$$

As $k$ is odd and $G_{1}^{\prime}\left(y_{k}\right)=y_{k}^{2}-\tau^{2}<0$, to fulfill $F_{1} G_{1}^{\prime}\left(y_{k}\right)>0$ it must be

$$
F_{1}\left(y_{k}\right)=y_{k}^{2}-\tau^{2}-\alpha \tau^{2}<0 \Leftrightarrow \alpha>y_{k}^{2} / \tau^{2}-1 .
$$

Now, as $G_{1}^{\prime}\left(y_{k+1}\right)=-y_{k+1}^{2}+\tau^{2}<0$ and one needs $F_{1} G_{1}^{\prime}\left(y_{k+1}\right)>0$, it follows

$F_{1}\left(y_{k+1}\right)=-y_{k+1}^{2}+\tau^{2}-\alpha \tau^{2}<0 \Leftrightarrow \alpha>-y_{k+1}^{2} / \tau^{2}+1$.

Next, it is considered the same situation with the other roots $y_{i}, y_{j}$ such that $0 \leq y_{i}<y_{k}<\tau<$ $y_{k+1}<y_{j}$ but in both cases one must take into account when the subscript $i$ is odd or even and the same with the subscript $j$.

i) Analysis for $y_{i}$

- $i$ odd: By means of (5) and (8) $G_{1}^{\prime}\left(y_{i}\right)=y_{i}^{2}-\tau^{2}<0$, so it should be $F_{1}\left(y_{i}\right)=y_{i}^{2}-\tau^{2}-\alpha \tau^{2}<0 \Leftrightarrow \alpha>$ 
$y_{i}^{2} / \tau^{2}-1$. The last inequality is valid due to $y_{i}<y_{k}$ and (A.3). So $\alpha>y_{k}^{2} / \tau^{2}-1>y_{i}^{2} / \tau^{2}-1$.

- $i$ even, $i \neq 0$ : Now, as $G_{1}^{\prime}\left(y_{i}\right)=-y_{i}^{2}+\tau^{2}>0$ and one needs $F G_{1}^{\prime}\left(y_{i}\right)>0$ follows $\alpha<-y_{i}^{2} / \tau^{2}+1$. The last inequality is valid if $\alpha<0$ due to $0<y_{i}<y_{k}<$ $\tau$ and $0<-y_{i}^{2} / \tau^{2}+1$.

- $i=0$ Now, as $G_{1}^{\prime}\left(y_{0}\right)=\tau^{2}>0$ and one needs $F_{1} G_{1}^{\prime}\left(y_{0}\right)>0$, it follows

$$
F_{1}\left(y_{0}\right)=\tau^{2}-\alpha \tau^{2}>0 \Leftrightarrow \alpha<1 .
$$

With $\alpha<0$, the condition (A.5) is already satisfied. ii) Analysis for $y_{j}$, where $y_{k}<\tau<y_{k+1}<y_{j}$. Again, two situations must be considered for $j$ : even or otherwise odd.

- $j$ even: By (8) results $G_{1}^{\prime}\left(y_{j}\right)=-y_{j}^{2}+\tau^{2}<0$, and this implies that $F_{1}\left(y_{j}\right)=-y_{j}^{2}+\tau^{2}-\alpha \tau^{2}<0 \Leftrightarrow \alpha>$ $-y_{j}^{2} / \tau^{2}+1$. This condition is satisfied due to $y_{k}<y_{j}$ and (A.4). So $\alpha>-y_{k+1}^{2} / \tau^{2}+1>-y_{j}^{2} / \tau^{2}+1$.

- $j$ odd: By (8) results $G_{1}^{\prime}\left(y_{j}\right)=y_{j}^{2}-\tau^{2}>0$, and this implies that $F_{1}\left(y_{j}\right)=y_{j}^{2}-\tau^{2}-\alpha \tau^{2}>0 \Leftrightarrow \alpha<$ $y_{j}^{2} / \tau^{2}-1$. This inequality is valid if $\alpha<0$ due to $0<y_{j}^{2} / \tau^{2}-1$ when $\tau<y_{k+1}<y_{j}$.

\section{Appendix B}

Proof. [Theorem 4] $\Longrightarrow$ (Necessary condition)

Case II)a): Let be $0<\bar{y}_{k}<\tau<\bar{y}_{k+1}$ where $k$ is odd. One must prove that $G_{2}$ (see (14)) has $4 k+2$ zeros in $[-2 k \pi+\pi / 2,2 k \pi+\pi / 2]$. Again $G_{2}(0)=0$. Just as in the proof of the necessary condition in I), the sign of $\delta$ is determined by the fact that the signs of $G_{2}\left(\bar{y}_{k}\right), G_{2}(\tau)$ and $G_{2}\left(\bar{y}_{k+1}\right)$ must alternate to guarantee the existence of roots of $G_{2}$ between the zeros of $F_{2}$. Thus $\delta>0, G_{2}\left(\bar{y}_{k}\right)>0$ and $G_{2}\left(\bar{y}_{k+1}\right)>0$ that yields $\delta<-\bar{y}_{k} / \tau+\tau / \bar{y}_{k}$ and $\delta<\bar{y}_{k+1} / \tau-\tau / \bar{y}_{k+1}$. So, there are two roots of $G_{2}$ in $\left[\bar{y}_{k}, \bar{y}_{k+1}\right]$. Besides, under these conditions, if $i<k$ and $i$ is odd then $G_{2}\left(\bar{y}_{i}\right)>0$, or if $i$ is even, $G_{2}\left(\bar{y}_{i}\right)<0$. Then, $G_{2}$ has one root in each interval $\left[\bar{y}_{i}, \bar{y}_{i+1}\right]$ as well as in $\left[y_{j}, y_{j+1}\right]$ if $j \geq k+1$ and altogether it has $4 k+2$ in $[-2 k \pi+\pi / 2,2 k \pi+\pi / 2]$. $\Longleftarrow$ (Sufficient condition)

Case II)a): By Theorem 2, it is necessary to set conditions to satisfy $F_{2}^{\prime} G_{2}(y)=F_{2}^{\prime}(y) G_{2}(y)<0$ for any root $y$ of $F_{2}$ given by (15) with $\gamma=1$. To prove this, the work is divided in two parts considering 1 ) $\breve{y}_{1,2}= \pm \tau$ and 2) $\bar{y}_{k}=(2 k-1) \pi / 2, k \in \mathbb{N}$.

Let $0<\bar{y}_{k}<\tau<\bar{y}_{k+1}$ and $\bar{y}_{k+1}=(2 k+1) \pi / 2$, where $k$ is odd.

1) Analysis for the roots $\check{y}_{1,2}= \pm \tau$.
Due to (14) and (17), results $F_{2}^{\prime}\left(\check{y}_{1,2}\right)=$ $\mp 2 \tau \cos \tau$ and $G_{2}\left(\check{y}_{1,2}\right)=-\delta \tau( \pm \tau)$, then the stability condition $F_{2}^{\prime} G_{2}(y)<0$ for the zeros of $F_{2}$, becomes $F_{2}^{\prime} G_{2}\left(\check{y}_{1,2}\right)=2 \delta \tau^{3} \cos \tau<0$. For $\bar{y}_{k}<\tau<$ $\bar{y}_{k+1}, \bar{y}_{k}=(2 k-1) \pi / 2$, with $k$ odd, as $\cos \tau<0$ then it must be $\delta>0$.

2) Analysis with the roots $\bar{y}_{k}$.

From (14) and (17), evaluating for $\bar{y}_{k}$ one has

$$
\begin{aligned}
F_{2}^{\prime}\left(\bar{y}_{k}\right) & =(-1)^{k}\left(-\bar{y}_{k}^{2}+\tau^{2}\right), \\
G_{2}\left(\bar{y}_{k}\right) & =(-1)^{k+1}\left(-\bar{y}_{k}^{2}+\tau^{2}\right)-\delta \tau \bar{y}_{k} .
\end{aligned}
$$

As $k$ is odd and $0<\bar{y}_{k}<\tau$ then $F_{2}^{\prime}\left(\bar{y}_{k}\right)=\bar{y}_{k}^{2}-\tau^{2}<$ 0 , so to fulfill $F_{2}^{\prime} G_{2}\left(\bar{y}_{k}\right)<0$ one needs

$$
G_{2}\left(\bar{y}_{k}\right)=-\bar{y}_{k}^{2}+\tau^{2}-\delta \tau \bar{y}_{k}>0 \Leftrightarrow \delta<-\bar{y}_{k} / \tau+\tau / \bar{y}_{k} .
$$

Now, as $F_{2}^{\prime}\left(\bar{y}_{k+1}\right)=-\bar{y}_{k+1}^{2}+\tau^{2}<0$ and one needs $F_{2}^{\prime} G_{2}\left(\bar{y}_{k+1}\right)<0$, it follows

$$
\begin{aligned}
G_{2}\left(\bar{y}_{k+1}\right) & =\bar{y}_{k+1}^{2}-\tau^{2}-\delta \tau \bar{y}_{k+1}>0 \\
& \Leftrightarrow \delta<\bar{y}_{k+1} / \tau-\tau / \bar{y}_{k+1} .
\end{aligned}
$$

Next, the same situation is considered with the roots $\bar{y}_{i}, \bar{y}_{j}$ such that $0<\bar{y}_{i}<\bar{y}_{k}<\tau<\bar{y}_{k+1}<\bar{y}_{j}$.

i) Analysis for $\bar{y}_{i}$ : it is necessary to take into account the cases where $i$ is odd or even.

- $i$ odd: By means of (17), as $F_{2}^{\prime}\left(\bar{y}_{i}\right)=\bar{y}_{i}^{2}-\tau^{2}<0$, and due to (14), it should be $G_{2}\left(\bar{y}_{i}\right)=-\bar{y}_{i}^{2}+\tau^{2}-$ $\delta \tau \bar{y}_{i}>0 \Leftrightarrow \delta<-\bar{y}_{i} / \tau+\tau / \bar{y}_{i}$. The last inequality is valid due to $\bar{y}_{i}<\bar{y}_{k}$ and (B.1) which implies $-\bar{y}_{k} / \tau+\tau / \bar{y}_{k}<-\bar{y}_{i} / \tau+\tau / \bar{y}_{i}$.

- $i$ even: Through (17) results $F_{2}^{\prime}\left(\bar{y}_{i}\right)=-\bar{y}_{i}^{2}+\tau^{2}>0$ so by (14) it must be $\delta>\bar{y}_{i} / \tau-\tau / \bar{y}_{i}$. Supposing $\delta>0$, the last inequality is true thanks to $0>\bar{y}_{i} / \tau-\tau / \bar{y}_{i}$ if $0<\bar{y}_{i}<\bar{y}_{k}<\tau$.

ii) Analysis for $\bar{y}_{j}$ : where $\bar{y}_{k}<\tau<\bar{y}_{k+1}<\bar{y}_{j}$. Again, two situations must be considered for $j$ : even or otherwise odd.

- $j$ even: Through (17) and (14) results $F_{2}^{\prime}\left(\bar{y}_{j}\right)=$ $-\bar{y}_{j}^{2}+\tau^{2}<0$, so $G_{2}\left(\bar{y}_{j}\right)=\bar{y}_{j}^{2}-\tau^{2}-\delta \tau \bar{y}_{j}>$ $0 \Leftrightarrow \delta<\bar{y}_{j} / \tau-\tau / \bar{y}_{j}$. This last condition is satisfied due to $\bar{y}_{k+1}<\bar{y}_{j}$ and (B.2) which implies $\bar{y}_{k+1} / \tau-\tau / \bar{y}_{k+1}<\bar{y}_{j} / \tau-\tau / \bar{y}_{j}$.

- $j$ odd: Taking into account (17) and (14) results $F_{2}^{\prime}\left(\bar{y}_{j}\right)=\bar{y}_{j}^{2}-\tau^{2}>0$, so $G_{2}\left(\bar{y}_{j}\right)=-\bar{y}_{j}^{2}+\tau^{2}-\delta \tau \bar{y}_{j}<$ $0 \Leftrightarrow \delta>-\bar{y}_{j} / \tau+\tau / \bar{y}_{j}$. The last inequality is true due to $\delta>0$ and $0>-\bar{y}_{j} / \tau+\tau / \bar{y}_{j}$ if $\tau<\bar{y}_{k+1}<\bar{y}_{j}$.

\section{References}

Allwright D. J. [1977] "Harmonic balance and the Hopf bifurcation," Math. Proc. of Cambridge Phil. Soc. 82, pp. 453-467. 
Åström, K. J. \& Hägglund, T. [1984] "Automatic tuning of simple regulators with specifications on phase and amplitude margins," Automatica 20(5), pp. 645-651.

Atherton, D. P. [1975] Nonlinear Control Engineering - Describing Function Analysis and Design, Van Nostrand Reinhold, London.

Bellman, R. \& Cooke, K. [1963] DifferentialDifference Equations, Academic Press, New York.

Bellman, R. \& Danskin, J. Jr. [1954] A Survey of the Mathematical Theory of Time-Lag, Retarded Control and Hereditary Processes, Report 256, The Rand Corporation, California.

Campbell, S. A. \& Bélair, J. [1999] "Resonant codimension two bifurcation in the harmonic oscillator with delayed forcing," Canadian Applied Mathematics Quarterly 7(3), pp. 217-238.

Campbell, S. A. \& LeBlanc, V. G. [1998] "Resonant Hopf-Hopf interactions in delay differential equations," J. Dyn. Differ. Equations 10(2), pp. 327-346.

Engelborghs, K., Luzyanina, T. \& Roose, D. [2002] "Numerical bifurcation analysis of delay differential equations using DDE-Biftool," $A C M$ Trans. Math. Software 28(1), pp. 1-21.

Gentile F. S., Moiola J. L. \& Paolini E. E. [2012] "On the study of bifurcations in delaydifferential equations: A frequency-domain approach," Int. J. Bifurcation and Chaos 22(6), pp. 1250137(1-15).

Gentile F. S., Itovich, G. R. \& Moiola J. L. [2018] "Resonant 1:2 double Hopf bifurcation in an oscillator with delayed feedback," Nonlinear Dyn. 91(3), pp. 1779-1789.
Itovich, G. R., Gentile F. S. \& Moiola J. L. [2018] "Stability analysis of a time delay system with a quadratic nonlinearity," Int. Symp. Nonlinear Theo. \& Appls. (NOLTA), Tarragona, Spain.

Mees, A. I. \& Chua, L. O. [1979] "The Hopf bifurcation theorem and its applications to nonlinear oscillations in circuits and systems," IEEE Trans. Circuits Syst. 26(4), pp. 235-254.

Mees, A. I. [1981] Dynamics of Feedback Systems, John Wiley \& Sons, Chichester, UK.

Moiola, J. L. \& Chen, G. [1996] Hopf Bifurcation Analysis: A Frequency-Domain Approach, Vol. 21, World Scientific, Singapore.

Pontryagin, L. S. "On the zeros of some elementary trascendental Functions," Amer. Math. Soc. Transl., Ser. 2, Vol. 1, 1955, pp. 95-110.

Prokop, R., Korbel, J. \& Matušů, R. [2012] "Autotuning principles for time-delay systems," WSEAS Trans Syst. 11(10), pp. 561-570.

Schei, T. S. [1994] "Automatic tuning of PID controllers based on transfer function estimation," Automatica 30(12), pp. 1983-1989.

Sipahi, R., Niculescu, S. I., Abdallah, C. T., Michiels, W. \& Gu, K. [2011] "Stability and stabilization of systems with time delay," IEEE Control Syst. Mag. 31(1), pp. 38-65.

Stépán, G. [1989] Retarded Dynamical Systems: Stability and Characteristic Functions, Longman, UK.

Tsypkin, Ya. Z. [1946] "Stability of systems with delayed feedback," Automat. Telemekh. 7, pp. 107-129 (also in MacFarlane, A. G. J. (Ed.) [1979] Frequency-Response Methods in Control Systems, IEEE Press, pp. 45-56). 